\title{
A rapid and durable response to cabozantinib in an osimertinib- resistant lung cancer patient with MET D1228N mutation: a case report
}

\author{
Yukun Kuang ${ }^{1,2 \#}$, Jiyu Wang ${ }^{1,2 \#}$, Peihang Xu ${ }^{1,2 \#}$, Yifan Zheng ${ }^{3 \#}$, Lihong Bai ${ }^{1,2}$, Xue Sun ${ }^{1,2}, Z_{i m u ~} i^{1,2}$, \\ Runjing Gan ${ }^{1,2}$, Huixia $\mathrm{Li}^{1,2}$, Zunfu Ke ${ }^{4}$, Kejing Tang ${ }^{1,2} \wedge$ \\ ${ }^{1}$ Division of Pulmonary and Critical Care Medicine, The First Affiliated Hospital, Sun Yat-sen University, Guangzhou, China; ${ }^{2}$ Institute of \\ Pulmonary Diseases, Sun Yat-sen University, Guangzhou, China; ${ }^{3}$ Department of Pharmacy, the First Affiliated Hospital, Sun Yat-sen University, \\ Guangzhou, China; ${ }^{4}$ Department of Pathology, The First Affiliated Hospital, Sun Yat-sen University, Guangzhou, China \\ \#These authors contributed equally to this work. \\ Correspondence to: Zunfu Ke. Department of Pathology, The First Affiliated Hospital, Sun Yat-sen University, No. 58, Zhongshan Er Lu, Guangzhou \\ 510080, China. Email: kezunfu@mail.sysu.edu.cn; Kejing Tang. Division of Pulmonary and Critical Care Medicine, The First Affiliated Hospital, \\ Sun Yat-sen University; Institute of Pulmonary Diseases, Sun Yat-sen University, No. 58, Zhongshan Er Lu, Guangzhou 510080, China. Email: \\ tangkj@mail.sysu.edu.cn.
}

\begin{abstract}
Osimertinib has efficacy superior to that of standard epidermal growth factor receptor (EGFR)tyrosine kinase inhibitors (TKIs) for the first-line treatment of patients with EGFR-mutant advanced non-small cell lung cancer (NSCLC). However, patients treated with osimertinib eventually acquire drug resistance. MET missense mutations have been demonstrated to mediate resistance to MET-TKIs, such as crizotinib. But the role of MET missense mutations in mediating EGFR TKI resistance is undefined. With the increasing use of next-generation sequencing (NGS) at diagnosis, many mechanisms of acquired resistance have been discovered in patients with activated tyrosine kinase receptors. Herein, we report the first case of MET D1228N mutation mediating acquired resistance to osimertinib in a MET TKI-naïve NSCLC. The patient with advanced lung adenocarcinoma harboring EGFR exon 19 deletion initially responded to osimertinib with progression-free survival (PFS) lasting 11 months and then developed resistance with an acquired mutation of MET D1228N. Subsequently, combination therapy of cabozantinib and osimertinib was administrated to the patient, and her clinical symptoms were rapidly relieved within one week with good tolerance. She remained on the combined treatment for 10 months. Finally, she achieved an overall survival (OS) of 25 months. Based on our findings, patient with MET D1228N mutant lung adenocarcinoma clinically benefited from combinatorial therapy of cabozantinib and osimertinib after osimertinib resistance.
\end{abstract}

Keywords: Lung adenocarcinoma; osimertinib resistance; MET D1228N mutation; cabozantinib; case report

Submitted Jul 11, 2021. Accepted for publication Aug 11, 2021.

doi: 10.21037/atm-21-3861

View this article at: https://dx.doi.org/10.21037/atm-21-3861

\section{Introduction}

The irreversible epidermal growth factor receptor tyrosine kinase inhibitor (EGFR-TKI), osimertinib, is a preferred first-line option for patients with EGFR-mutant metastatic non-small cell lung cancer (NSCLC) (1). With the wide application of osimertinib, its resistance mechanisms

^ ORCID: 0000-0002-5454-4666. 

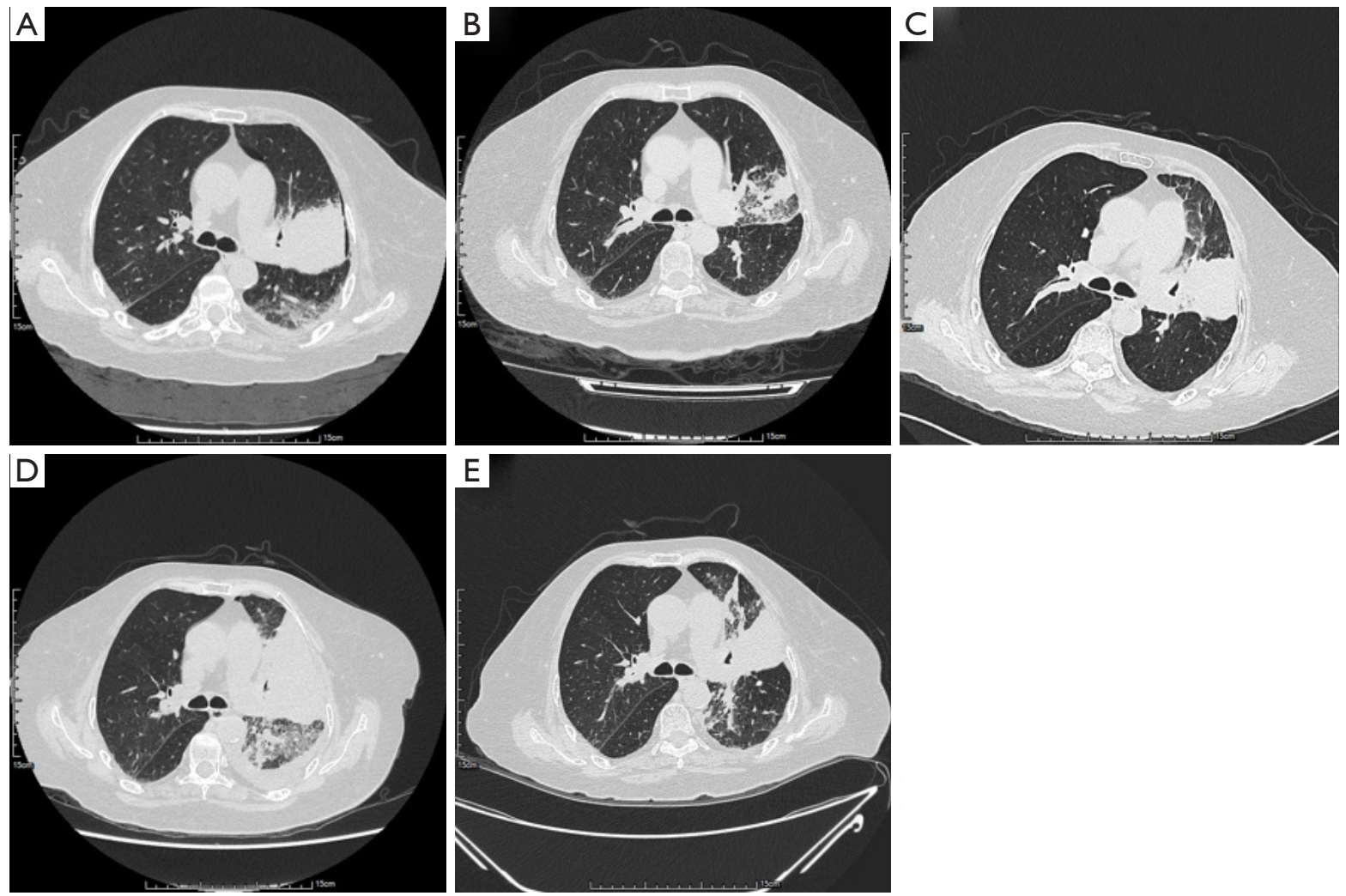

Figure 1 Chest CT images of an advanced NSCLC patient with EGFR exon 19 deletion. (A) At baseline. (B) Confirmed PR after 3 months of osimertinib. (C) Asymptomatic PD after 11 months of osimertinib. (D) Left lung lesion growth with left pleural effusion. (E) Immediate remission after the addition of cabozantinib. CT, computed tomography; NSCLC, non-small cell lung cancer; EGFR, epidermal growth factor receptor; PR, partial response; PD, progressive disease.

have been well elucidated, among which the activation of the MET pathway via $M E T$ gene activation and amplification is one of the most common bypass pathway mechanisms (2). Combining c-Met inhibitors, such as crizotinib, with osimertinib has been found to be effective in osimertinib-resistant EGFR-mutated NSCLC patients harbouring MET amplification (3). On the other hand, acquired mutations, including those at positions Y1230 and D1228, can occur over long-term exposure to MET inhibitors (4). However, bypass activation of MET by missense mutation has not been reported to mediate the resistance to EGFR TKIs thus far. In this study, we report the first case of MET D $1228 \mathrm{~N}$ mutation as the resistant mechanism to osimertinib in a MET TKI-naïve lung adenocarcinoma patient who subsequently benefited from the combinational therapy of EGFR and MET TKIs. We present the following case in accordance with the CARE reporting checklist (available at https://dx.doi. org/10.21037/atm-21-3861).

\section{Case presentation}

An 81-year-old female patient presented to the hospital in May 2018 with symptoms of productive cough and shortness of breath for several months. A chest computed tomography (CT) scan showed a $58 \mathrm{~mm} \times 62 \mathrm{~mm}$ nodule in the left lung with multiple small nodules, enlarged mediastinal lymph nodes, and pleural effusions (Figure 1A). She was diagnosed with stage IV lung adenocarcinoma and capture-based nextgeneration sequencing (NGS) with a panel of 168 cancerrelated genes (Lung Plasma, Burning Rock, Guangzhou, China) was conducted with a lung biopsy specimen. EGFR c.2236_2250del (p.E746_A750del) mutation (abundance $11.52 \%)$ was identified, and she thus received osimertinib (80 $\mathrm{mg}, \mathrm{qd})$ as the first-line therapy. Her symptoms improved rapidly, and no significant adverse events were observed. A chest CT evaluation 12 weeks later showed a partial response (PR) and the lung lesion significantly decreased $(38 \mathrm{~mm} \times 22 \mathrm{~mm}$; Figure $1 B)$. After 11 months 


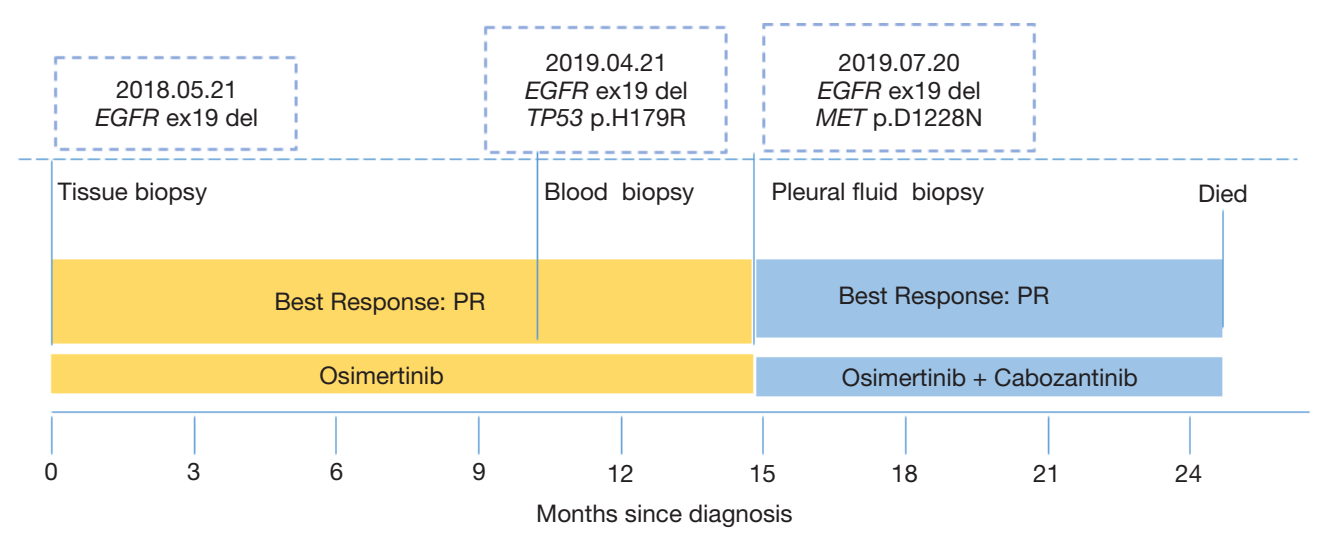

Figure 2 Timeline and duration of each treatment.

of disease control, a CT scan showed an enlarged left lung mass (66 $\mathrm{mm} \times 50 \mathrm{~mm}$; Figure 1C). Ultra-deep plasmaderived circulating tumor DNA (ctDNA) NGS revealed EGFR E746_A750del (abundance 0.54\%) and TP53 H179R mutation (abundance $1.32 \%$ ). The patient presented asymptomatic disease progression (PD) and refused chemotherapy, so she continued osimertinib treatment (80 mg, qd). She was readmitted to the hospital with progressive aggravating symptoms in July 2019. Chest CT showed that the mass in the upper lobe of the left lung was enlarged and densified significantly with increased left pleural effusion $(97 \mathrm{~mm} \times 67 \mathrm{~mm}$; Figure $1 D)$, indicating PD with serious clinical symptoms. Along with intensive respiratory support, the patient received ultrasoundguided pleural puncture and catheterization. Hydrothorax exudate NGS showed EGFR E746_A750 deletion (abundance $4.95 \%$ ) and MET D1228N missense mutation (abundance $3.86 \%$ ). Treatment was subsequently changed to cabozantinib (40 mg, qd) plus osimertinib (80 mg, qd), and she experienced an overall improvement in her clinical symptoms within 1 week. Re-examination of CT 10 days later revealed that the primary lesion of the left lung had dramatically shrunk $(65 \mathrm{~mm} \times 65 \mathrm{~mm}$; Figure $1 E)$, and the efficacy was evaluated as PR. After discharge, the patient continued the combined treatment of cabozantinib and osimertinib, and no other adverse events occurred except for grade 2-3 diarrhea. Due to personal reasons, the patient did not return regularly. She took cabozantinib and osimertinib until her symptoms worsened again in May 2020. Finally, she died 1 month later with an overall survival (OS) of 25 months. The overall process of diagnosis and treatment is shown in Figure 2. All procedures performed in studies involving human participants were in accordance with the ethical standards of the institutional and/or national research committee(s) and with the Helsinki Declaration (as revised in 2013). Written informed consent was obtained from the patient for publication of this case report and accompanying images. A copy of the written consent is available for review by the editorial office of this journal.

\section{Discussion}

The acquired resistance mechanisms to first-line or secondline osimertinib therapy have been illustrated, including $E G F R$-dependent and bypass resistance mechanisms. One of the most frequent causes of bypass pathway activation is $M E T$ amplification. Furthermore, oncogenic alteration $M E T$ exon 14 skipping can induce resistance to osimertinib, which has been confirmed in EGFR-mutated cell models (5). However, mutations in the MET tyrosine kinase domain have rarely been found in NSCLC as a secondary event resulting from exposure to prior therapies, such as TKIs (6).

In a recent case reported by Kang et al., one patient benefited from the combination of osimertinib and cabozantinib after simultaneous development of secondary MET mutations upon crizotinib treatment (7). A combination of osimertinib and trastuzumab emtansine ( $\mathrm{T}-$ DM1), a conjugate of the monoclonal antibody trastuzumab (Herceptin) and the cytotoxic agent DM1, was reported to delay and overcome osimertinib resistance in T790Mpositive EGFR-mutated NSCLC cell lines that gained HER2 amplification (8).

In our case, MET D1228N mutation, a known resistance mutation to type I MET inhibitors, was acquired after firstline osimertinib failure without any prior exposure to MET inhibitors. Given that D1228N shows an increased level of 
tyrosine phosphorylation with respect to wild-type $M E T$ (9), we consider it could mediate the resistance to EGFR-TKI osmertinib.

MET D1228N mutation shows resistance to type I MET inhibitors, such as crizotinib and capmatinib, but displays sensitivity to type II inhibitors, such as cabozantinib in vitro (10). However, in clinical settings, the sensitivity of MET D $1228 \mathrm{~N}$ to MET inhibitors is equivocal. MET D1228N bypass activation was identified as the potential mechanism to crizotinib resistance in a CD74-ROS1 fusion lung cancer patient. Second-line cabozantinib brought significant improvement and 3 months of progressionfree survival for the patient (11). In another report, lung cancer patient who was resistant to osimertinib due to MET amplification received crizotinib and osimertinib therapy (7). Upon confirmation of PD, novel acquired MET mutations, D1228N/Y, Y1230H, and D1231Y were detected (7). The treatment was changed from crizotinib to cabozantinib, and, after the combinatorial treatment, repeat dynamic liquid biopsy revealed that all $M E T$ point mutations were undetectable, except for D1228N (7). The allelic fraction of this mutation increased compared to the baseline, suggesting the subclonal lesion bearing this variant was nonresponsive to cabozantinib (7). MET D $1228 \mathrm{~N}$ was also reported to be an acquired resistance mutation to crizotinib in an $M E T$-amplified advanced triple-negative breast cancer patient. She was treated with cabozantinib for 7 weeks followed by rapid progression (12).

Although the efficacy of cabozantinib to MET D1228N mutation remains inconclusive, we treated our patient with cabozantinib and osimertinib after consulting with the patient and her family. Her clinical symptoms were rapidly relieved with 1 week, and she obtained an OS of 25 months. The limitation of this case was loss to follow up during late course of treatment, and the PFS was not available. Collectively, the effectiveness of cabozantinib against MET D1228N mutation may differ among patients due to the type of cancer, performance status, and other unknown tumor-intrinsic characteristics. Further investigations are still needed to elucidate the resistance mechanism of acquired MET D1228N mutations to develop more efficacious MET inhibitors.

\section{Conclusions}

Our study was the first to report that MET D1228N mutation can also mediate resistance to first-line osimertinib therapy. Patient with MET D1228N mutation clinically benefited from combinatorial therapy of cabozantinib and osimertinib after osimertinib resistance.

\section{Acknowledgments}

Funding: This work was supported by grants from the Guangzhou Science and Technology Planning Program [No. 202002030023]; and Natural Science Foundation of Guangdong Province [18zxxt17].

\section{Footnote}

Reporting Checklist: The authors have completed the CARE reporting checklist. Available at https://dx.doi. org/10.21037/atm-21-3861

Conflicts of Interest: All authors have completed the ICMJE uniform disclosure form (available at https://dx.doi. org/10.21037/atm-21-3861). The authors have no conflicts of interest to declare.

Ethical Statement: The authors are accountable for all aspects of the work in ensuring that questions related to the accuracy or integrity of any part of the work are appropriately investigated and resolved. All procedures performed in studies involving human participants were in accordance with the ethical standards of the institutional and/or national research committee(s) and with the Helsinki Declaration (as revised in 2013). Written informed consent was obtained from the patient for publication of this case report and accompanying images. A copy of the written consent is available for review by the editorial office of this journal.

Open Access Statement: This is an Open Access article distributed in accordance with the Creative Commons Attribution-NonCommercial-NoDerivs 4.0 International License (CC BY-NC-ND 4.0), which permits the noncommercial replication and distribution of the article with the strict proviso that no changes or edits are made and the original work is properly cited (including links to both the formal publication through the relevant DOI and the license). See: https://creativecommons.org/licenses/by-nc-nd/4.0/.

\section{References}

1. Soria JC, Ohe Y, Vansteenkiste J, et al. Osimertinib in Untreated EGFR-Mutated Advanced Non-Small-Cell 
Lung Cancer. N Engl J Med 2018;378:113-25.

2. Ramalingam SS, Yang JC, Lee CK, et al. Osimertinib As First-Line Treatment of EGFR Mutation-Positive Advanced Non-Small-Cell Lung Cancer. J Clin Oncol 2018;36:841-9.

3. Leonetti A, Sharma S, Minari R, et al. Resistance mechanisms to osimertinib in EGFR-mutated non-small cell lung cancer. Br J Cancer 2019;121:725-37.

4. Heist RS, Sequist LV, Borger D, et al. Acquired Resistance to Crizotinib in NSCLC with MET Exon 14 Skipping. J Thorac Oncol 2016;11:1242-5.

5. Suzawa K, Offin M, Schoenfeld AJ, et al. Acquired MET Exon 14 Alteration Drives Secondary Resistance to Epidermal Growth Factor Receptor Tyrosine Kinase Inhibitor in EGFR-Mutated Lung Cancer. JCO Precis Oncol 2019;3:PO.19.00011.

6. Engelman JA, Jänne PA. Mechanisms of Acquired Resistance to Epidermal Growth Factor Receptor Tyrosine Kinase Inhibitors in Non-Small Cell Lung Cancer. Clinical Cancer Research 2008;14:2895-9.

7. Kang J, Chen HJ, Wang Z, et al. Osimertinib and Cabozantinib Combinatorial Therapy in an EGFRMutant Lung Adenocarcinoma Patient with Multiple MET Secondary-Site Mutations after Resistance to

Cite this article as: Kuang Y, Wang J, Xu P, Zheng Y, Bai L, Sun X, Li Z, Gan R, Li H, Ke Z, Tang K. A rapid and durable response to cabozantinib in an osimertinib-resistant lung cancer patient with MET D1228N mutation: a case report. Ann Transl Med 2021;9(16):1354. doi: 10.21037/atm-21-3861
Crizotinib. J Thorac Oncol 2018;13:e49-e53.

8. La Monica S, Cretella D, Bonelli M, et al. Trastuzumab emtansine delays and overcomes resistance to the thirdgeneration EGFR-TKI osimertinib in NSCLC EGFR mutated cell lines. J Exp Clin Cancer Res 2017;36:174.

9. Bardelli A, Longati P, Gramaglia D, et al. Uncoupling signal transducers from oncogenic MET mutants abrogates cell transformation and inhibits invasive growth. Proc Natl Acad Sci U S A 1998;95:14379-83.

10. Engstrom LD, Aranda R, Lee M, et al. Glesatinib Exhibits Antitumor Activity in Lung Cancer Models and Patients Harboring MET Exon 14 Mutations and Overcomes Mutation-mediated Resistance to Type I MET Inhibitors in Nonclinical Models. Clin Cancer Res 2017;23:6661-72.

11. Wang Y, Chen Z, Han X, et al. Acquired MET D1228N Mutations Mediate Crizotinib Resistance in Lung Adenocarcinoma with ROS1 Fusion: A Case Report. Oncologist 2021;26:178-81.

12. Parsons BM, Meier DR, Richmond CS, et al. Acquisition of Cabozantinib-Sensitive MET D1228N Mutation During Progression on Crizotinib in MET-Amplified Triple-Negative Breast Cancer. Clin Breast Cancer 2020;20:e433-8. 